\title{
ACERCA DE LA RELACIÓN GENEALÓGICA DE LAS LENGUAS LENCAS Y LAS LENGUAS MISUMALPAS
}

\begin{abstract}
RESUMEN
En este artículo se realiza un estudio acerca de las relaciones genealógicas de dos familias lingüísticas de Centroamérica: la lenca y la misumalpa, mediante la aplicación del método comparativo.
\end{abstract}

Adolfo Constenla Umaña

\begin{abstract}
In this paper, a study about the genealogical relations of two linguistics families of Central America is made: the lenca and the misumalpa by applying the comparative method.
\end{abstract}

\section{Introducción}

Este estudio trata sobre las relaciones genealógicas de dos familias lingüísticas centroamericanas habladas en territorios contiguos: la lenca y la misumalpa.

La familia lenca estaba integrada por dos lenguas: el lenca de Honduras y el lenca de El Salvador, cuya separación data, según la aplicación de la glotocronología hecha en este estudio, de hace unos 2300 años. Ocupaba (Chapman 1978) la porción de El Salvador situada al este del río Lempa, así como algunos enclaves situados al oeste del mismo (Fowler 1989: 64), y la mayor parte del centro de Honduras (departamentos del Valle, de la Paz, Intibucá, Lempira, Comayagua; sur del Departamento de Santa Bárbara, centro y sur del Departamento de Francisco Morazán y este del Departamento de Choluteca).

Arguedas Cortés (1987) aplicó el método comparativo y reconstruyó el sistema fonológico del protolenca y 89 rubros léxicos de esta protolengua. Posteriormente se han aportado 22 nuevos conjuntos de cognados y observaciones sobre algunos rasgos sintácticos que se le pueden atribuir al protolenca (Constenla Umaña 1991: 19 y 186).

En cuanto a las relaciones externas de la familia lenca, se han propuesto con las lenguas mixe-zoques (Lehmann 1920: 641-48; Mason 1943: 241), con las xincas (Lehmann 1920: 767, Swadesh 1958: 133-4), con las anteriores, las misumalpas, el paya y las chibchas en general (Schmidt 1926 : 206-8, Jijón y Caamaño 1943 : 11, Greenberg 1987: 382), con las utoaztecas (Escalante y Faier 1959) y con las mayas (Wyllys 1979).

De todos estos autores, aparentemente sólo Lehmann (para el caso de la relación con el xinca) Wyllys y Greenberg intentaron aducir indicios. En todos estos casos o no se dan 
indicios o los que intenta aportar están en contradicción con los métodos apropiados de la lingüística diacrónica (véanse, por ejemplo, los comentarios de Campbell 1979 : 961-2, Constenla Umaña 1991:24, 1993: 88-94).

La familia misumalpa ocupaba la mayor parte del territorio del territorio de Nicaragua, excluida la vertiente pacífica y la porción de la vertiente caribe situada al sur de la laguna de Blufields y al este del Lago de Nicaragua, y se extendía hasta los departamentos hondureños de El Paraíso, Choluteca y Gracias a Dios. En el oriente de El Salvador había un enclave misumalpa: la lengua cacaopera. Las otras lenguas que integran esta familia son: el matagalpa, el misquito, el sumo y el ulua. La presencia de topónimos matagalpas en Chinandega (véase Constenla Umaña 1994:195 ) es un indicio de que la parte norte del litoral pacífico nicaragüense hubiera sido también territorio misumalpa antes de su ocupación por pueblos de origen mesoamericano, como los chorotegas y los nicaraos.

Constenla Umaña $(1987,1991)$ llevó a cabo la reconstrucción del sistema fonológico, de algunos rasgos morfológicos y sintácticos y de 94 rubros léxicos del protomisumalpa. El análisis aglomerativo (cluster analysis) de las matrices de porcentajes de cognados obtenidos por Moreira González en 1986 (Constenla Umaña 1991) dividió a la familia en dos ramas: el misquito y una, que a su vez incluye dos subgrupos: el matagalpa-cacaopera y el sumo-ulua.

Por lo que respecta a sus relaciones externas, la familia misumalpa se ha considerado desde Lehmann en adelante como parte de lo que se ha denominado filo macrochibcha, con todas las relaciones que esto implica según el autor de que se trate. Hasta el momento ninguna de ellas ha sido probada.

En este trabajo se darán pruebas, por medio de la aplicación del método comparativo, de que las familias en cuestión en efecto están relacionadas genealógicamente. Además, se lleva a cabo un estudio glotocronológico con la finalidad de determinar la fecha aproximada en que el protolenca-misumalpa inició su división en protolenca y protomisumalpa.

\section{Conjuntos de cognados lenca-misumalpas}

En 1991 (Constenla Umaña 1991: 186) ofrecí una lista de 64 conjuntos de cognados lenca-misumalpas, que con excesiva cautela califiqué de 'aparentes'. Posteriormente he encontrado 28 más. Este número sin duda se ampliará cuando se tomen en cuenta todos los materiales del lenca de Honduras (hay por lo menos tres vocabularios importantes que no he podido consultar).

Los datos del lenca de El Salvador se han tomado de Lehmann (1920), Cambell 1976 y 1979, y Del Río Urrutia (1985); en todos los casos, los datos se representan de acuerdo con la fonematización propuesta por esta última autora. En el caso del lenca de Honduras, se emplearon los léxicos incluidos en Lehmann (ibídem), Campbell y otros (1978), Herranz (1987) y Arguedas Cortés (1987); todos los rubros citados se redujeron a la fonematización propuesta por esta última autora. Para las lenguas misumalpas se usaron los léxicos contenidos en Lehmann (ibídem), el diccionario sumo de von Houwald (1980), el diccionario ulua del CIDCA y el ITM (1988), el diccionario misquito del CIDCA (1986), el léxico cacaopera reunido en Bertoglia Richards (1988) y datos míos de misquito. 
Abreviaturas

$\begin{array}{llll}\text { Lenca de El Salvador } & \text { LS } & \text { Misquito } & \text { MIS } \\ \text { Lenca de Honduras } & \text { LH } & \text { Sumo } & \text { SU } \\ \text { Matagalpa } & \text { MA } & \text { Ulua } & \text { UL } \\ \text { Cacaopera } & \text { CA } & & \end{array}$

1. abuela titi (CA), titị̣ (SU), teteh (LS), texta (LH)

2. achiote awal (SU), awal (UL), awalk'e (LS)

3. agua was 'lluvia' (MA), was (SU), was (UL), wal (LS), was (LS)

4. aguacate sjal (MA), sjal (CA), sikja (MIS), sjal (LH), jik'a (LS)

5. alacrán wakurus (SU), wa:kurus (UL), wạ̣kirini (LH)

6. amarrar wiri- (CA), wilk- (MIS), war- (LS)

7. anciano(a) kukus (CA), kukự (SU), kuka (MIS), koko (LS)

8. ayote iwa (CA), îwä̀ (MIS),ts'iwạ̣ (LS), ewa (LH)

9. aquí nãhãra (MIS), nanum (LS), nawah (LH)

10.blanco sahu (CA), joko (LS), soko (LH)

11. bueno jamni (SU), jamka (UL), jamni (MIS), jam (LS), sa (LH)

12. búho: $\quad$ iskiri (CA), iskiri (SU), iskri (MIS), iskrip'up'u (LS)

13. cabeza tuna (SU), tuni (UL), troh $(\mathrm{LH})$

14. carne wina (MIS), waja (LS)

15. cola wajka (MIS), wah (LH)

16. comer kas- (SU), kas- (UL), kor- (LH)

17. comprar liwan 'dinero' (CA), lihwan 'dinero' (UL), liwa- (LS), liwa-(LH)

18. correr ir- (SU), i:ra- (UL), ir- (LH)

19. cortar dak- (SU), dak- (UL) t'ih- (LS), taj- (LH)

20. cuatro arunka (SU), arunka (UL), aria (LH)

21. cubrir tụ̣- (UL), tum 'cubierto' (LH)

22. cuerda kan (CA), kan (LH)

23. dar ja- (UL), ja-b- (MIS), jar- (LH)

24. decir ajs 'hablar' (MIS), aj- (LS), aj- (LH)

25. diente nini- (SU), nini (UL), neh (LS), nek (LH)

26. chupar su- (SU), ts'u- (LS), su- (LH)

27. dónde ankat (SU), a:nira (MIS), anụ̣ (LS)

28. dulce damni (SU), damka (UL), damni (MIS), tọ̣ka (LS)

29. elequeme tukrụ̣ (SU), tukrụ̄ (UL), tukrụ̣ (MIS), t'uku (LS)

30. en $\quad-m(\mathrm{CA})$, ap (LH)

31. en -kaw, -kat (SU), -kaw, -kat (UL), -kat 'hasta' (MIS), k'ati (LS)

32. este irahi (CA) inaga (LS)

33. este nãhã (MIS), na (LH)este ( cf. na-num 'aquí', LS)

34. estrella slilma (MIS), sirih (LS), siri (LH)

35. excremento ba (SU), ba (UL), po- 'defecar' (LS)

36. extremidad kari 'brazo' (CA), kal 'pie' (SU), kal 'pie' (UL), klahkla 'brazo' (MIS), kal ‘brazo/aleta' (LS) 
37. frío sạ̣ni (SU), ts'ana (LS)

38. guacal kahami (MIS), k'ama (LS)

39. guacamaya awa (SU), awa (UL), awsa (MIS), ewa (LS), ewa (LH)

40. guatuza kiki (CA), kjaki (MIS), keke (LS)

41. guayabo tur (CA), tororo (LS)

42. hoja waha (MIS), wala (LH)

43. hombre misa (MA), misil (CA), misu (LH)

44. ir

wa- (CA), wat- 'caminar' (UL), wa- 'ir', wap- 'caminar' (MIS), o- 'ir, caminar' (LS), u- 'ir, caminar' (LH)

45. jaguar nama (MA), namas (CA), nawah (SU), nawah (UL), limi/lamja (MIS), lepa (LS), lepa (LH)

46. jocote ura (CA), walak (SU), walak (UL), muraka (LS), murak (LH)

47. laurel sum (SU), sum(UL), sum (MIS), jumam (LS)

48. lavar saka- (CA), ts'ih- (SU), sak (UL)

49. madre amis (MA), ami (CA), mina (LH)

50. maíz ajma (MA), ajma (CA), ama (SU), am (UL), ima (LS), ama (LH)

51. mapachín suksuk (SU), suksuk (MIS), susumi (LH)

52. miel ala (CA), walạ̣kka (SU), walạ̣ka (UL), walaṇka 'abeja tacaní'

(MIS), wehle (LS)

53. mosca kî̉kas (MIS), kuku (LH)

54. montaña karan (CA), kotañ (LS), kotạ̣ (LH)

55. morder kas- (SU), kas- (UL), kaj- (LH)

56. nariz nam-n (MA), nam-n (CA), nam- (SU), nan-tak (UL), nepkuru(LS), nepsek (LH)

57. negro siksa (MIS) sị̣ka (LS), siri (LH)

58. niño misa (CA), mesi (LH)

59. nube amu (CA), mukus (SU), mukus (UL), mukus (MIS), mo 'humo' (LS)

60. olomina urụ̄ni (CA), orum (LS)

61. ombligo klua (MIS), k'ul (LS)

62. padre

63. pequeño

wapa (CA), papaṇ (SU), papañ (UL), papa (LH)

64. perico

65. perro

66. pesado

țiki (CA), sirpi (MIS), ts'irijki (LS)

ilik (UL), kili (LH)

sulu (SU), sulu (UL), jul (MIS), juju (LS), suj (LH)

67. piedra

tihni/tikini (SU) ti:ka (UL), t'ili (LS), tiliba (LH)

(LH)

68. pita ki (SU), ki (UL), kipla 'peña', kisa 'pedernal' (MIS), ke (LS), ke

69. podrirse

kami (CA), kara (MIS), ka (LS)

70. pozol

71. pueblo

72. río

duraka (CA), dutla (SU), dutạ̣ (UL), lukawa (LS)

musu (MA), mujuwa (LS)

wina (CA), wiran (LH)

73. sangre

awala (MIS), wara (LS), wara (LH)

74. sardina

aru (CA), a (SU), a-was (UL), ala (LS)

75. seco

sajak (CA), ¡aja (LS)

sama (CA), sawa (LH) 


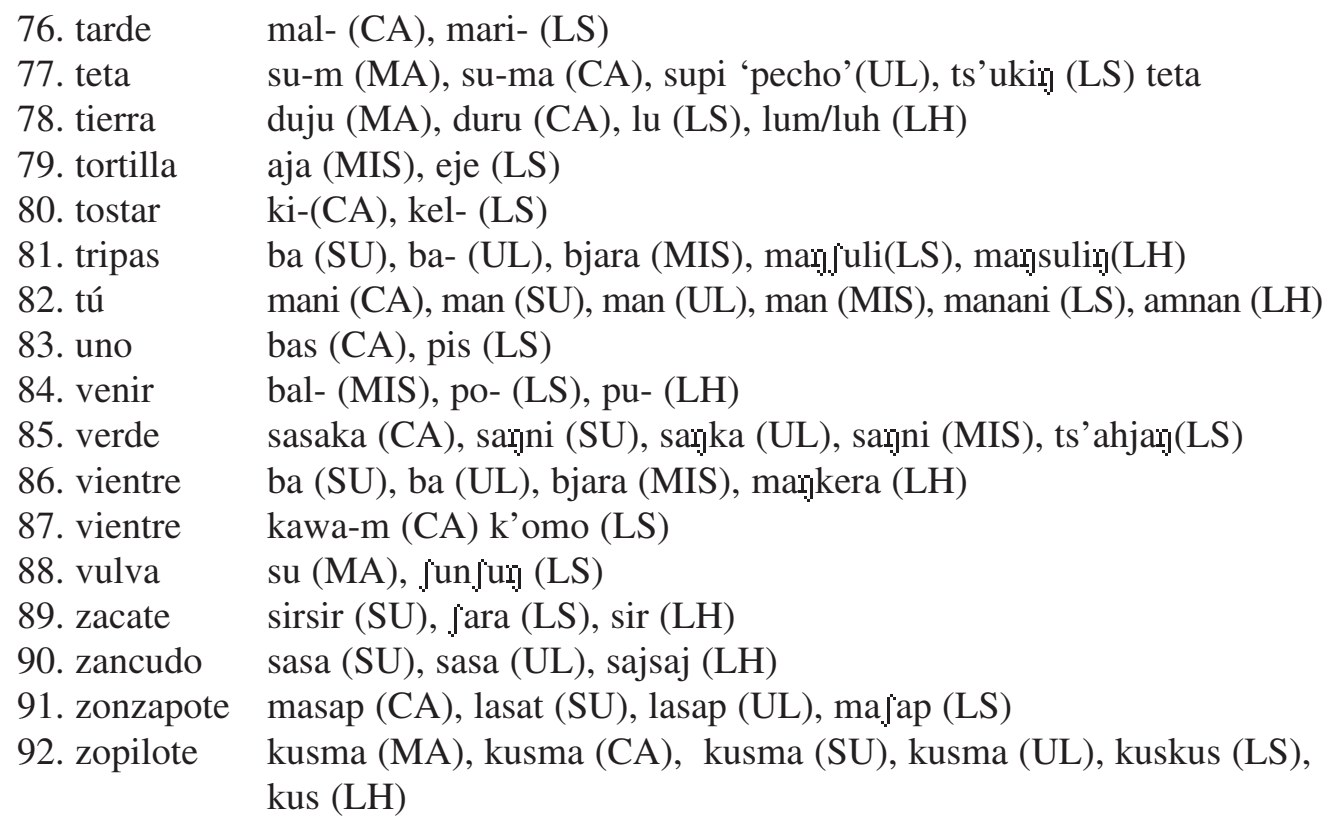

\section{Correspondencias fonológicas}

Como se puede observar de manera inmediata, en los datos se da un número importante de correspondencias fonológicas entre las lenguas lencas y las misumalpas que, a mi juicio, comprueban el parentesco entre las dos agrupaciones. No considero, sin embargo, estar todavía en condiciones de reconstruir el sistema fonemático de la protolengua, pues el número de cognados parece ser todavía insuficiente como para poder reconocer apropiadamente, por ejemplo, los entornos condicionantes de probables casos de escisión. A continuación enumero las principales correspondencias observadas, citándolas, siempre que sea posible, por los protofonemas entre los cuales se da la correspondencia, de acuerdo con las reconstrucciones disponibles de los subantepasados protolenca (PL) y protomisumalpa (PM) (Arguedas Cortés 1987, Constenla Umaña 1987):

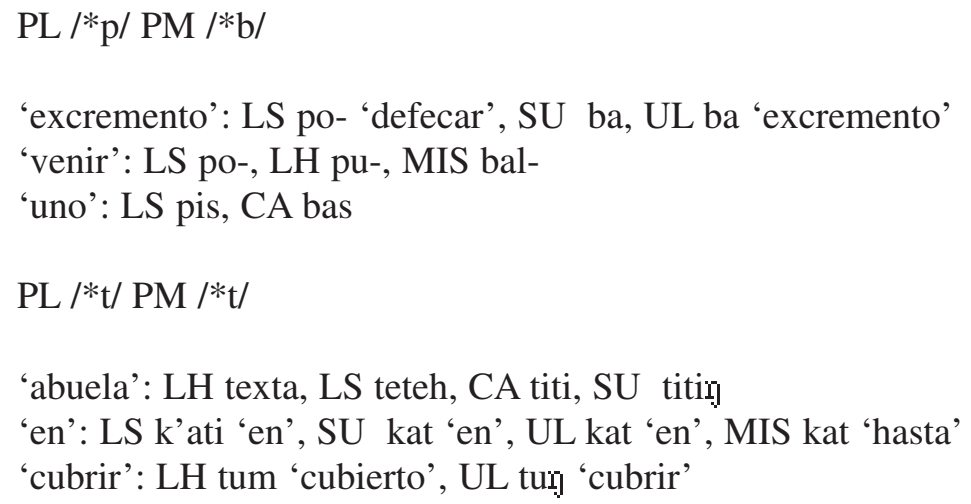




\section{$\mathrm{PL} / * \mathrm{t} ' / \mathrm{PM} / * \mathrm{t} /$}

'elequeme': LS t'uku, SU tukruṇ, UL tukruṇ, MIS tukrự 'pesado': LS t'ili, LH tiliba, SU tihni/tikini, UL ti:ka

\section{$\mathrm{LS} / \mathrm{l} / \mathrm{PM} / * \mathrm{~d} /$}

'podrirse': LS lukawa, CA duraka, SU dutla, UL dutaṇ 'tierra': LS lu, MA duju, CA duru

$\mathrm{PL} / * \mathrm{k} / \mathrm{PM} / * \mathrm{k} /$

'anciano(a)': LS koko, CA kukus, SU kukự, MIS kuka 'piedra': LH ke, LS ke, SU ki, UL ki; MIS kipla 'peña', kisa 'pedernal' 'extremidad': LS kal 'brazo/aleta', CA kari 'brazo', SU kal 'pie', UL kal 'pie', MIS klahkla 'brazo'

'jocote': LH murak, LS muraka, CA ura, SU walak, UL walak

$\mathrm{LS} / \mathrm{k} / \mathrm{PM} / * \mathrm{k} /$

'guacal': LS k'ama, MIS kahami

'ombligo':LS k'ul, MIS klua

'en': LS k'ati ‘en', SU kat 'en', UL kat 'en', MIS kat 'hasta'

'vientre': LS k'omo, CA kawa-m

$\mathrm{PL} / * \mathrm{ts}^{\prime} / \mathrm{PM} / * \mathrm{~s} /$

'frío': LS ts'ana, SU sanni

'pequeño': LS ts'irijki, MIS sirpi

'lavar':LH sak-, LS ts'ih-, CA saka-, MIS sik-ba-

'teta, chupar': LS ts'u- 'chupar', ts'ukiṇ 'teta', MA su-m 'teta', CA su-m 'teta', SU su- 'chupar', UL supi 'teta'

$\mathrm{PL} / * \mathrm{~s} / \mathrm{PM} / \mathrm{s} /$

'zonzapote': LS majap, CA masap, SU lasat, UL lasap

'laurel': LS jumam, SU sum, UL sum, MIS sum

'hombre': LH misu (misi 'muchacho'), MA misa, CA misil (misa 'muchacho')

'zacate': LH sir, LS jara, SU sirsir

'zopilote': LH kus, LS kuskus, MA kusma, CA kusma, SU kusma, UL kusma

$\mathrm{PL} / * 1 / \mathrm{PM} / * 1 /$ 
'perico': LH kili, UL ilik

‘ombligo': LS k'ul, MIS klua

'miel': LS wehle 'miel', CA ala 'miel', SU walạ̣ka 'abeja tacaní', UL walạ̣kka

'abeja tacaní', MIS walaṇka 'abeja tacaní'

'comprar', 'dinero': LH liwa- 'comprar', LS liwa- 'comprar', CA liwan 'dinero', UL lihwan 'dinero'

$\mathrm{PL} / * \mathrm{j} / \mathrm{PM} / * \mathrm{j} /$

'dar': LH jar-, UL ja:-

'tortilla': LH eje, MIS aja 'maíz'

'llorar': LS kaj-, SU kajd-Úajd-, UL ajd-

$\mathrm{PL} / *_{\mathrm{W}} / \mathrm{PM} / *_{\mathrm{W}} /$

'agua': LH was, LS wal, MA was 'lluvia', SU was, UL was

'guacamaya': LH ewa, LS ewa, SU awa, UL awa, MIS awsa

'pueblo': LH wiran, CA wina

‘río': LH wara LS wara, MIS awala

$\mathrm{PL} / * \mathrm{~m} / \mathrm{PM} / * \mathrm{~m} /$

'guacal': LS k'ama, MIS kahami

'laurel': LS Jumam, SU sum, UL sum, MIS sum

'madre': LH mina, MA amis, CA ami

'maíz': LH ama, LS ima, MA ajma, CA ajma, SU ama, UL am

$\mathrm{PL} / * \mathrm{n} / \mathrm{PM} / * \mathrm{n} /$

'nariz': LH nepsek, LS nepkuru, MA nam-n, CA nam, SU nạ̣-tak, UL nạ̣-tak

'este': LH na, LS na-num 'aquí', MIS naha

'diente': LH nek, LS neh, SU nini, UL nini

‘frío': LS ts'ana, SU saṇni

$\mathrm{PL} / * \mathrm{i} / \mathrm{PM} / * \mathrm{i} /$

'correr': LH ir-, UL i:ra-

'madre': LH mina, MA amis, CA ami

'búho’: LS iskrip’up'u, CA iskiri, SU iskiri, MIS iskri

‘pequeño': LS ts'irijןki, MIS sirpi

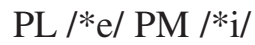

'diente': LH nek, LS neh, SU nini, UL nini 
'abuela': LH texta, LS teteh, CA titi, SU titị̄

'guatuza': LS keke, CA kiki, MIS kjaki

'piedra': LH ke, LS ke, SU ki, UL ki; MIS kipla 'peña', kisa 'pedernal'

$\mathrm{PL} / * \mathrm{e} / \mathrm{PM} / * \mathrm{a} /$

'guacamaya': LH ewa, LS ewa, SU awa, UL awa, MIS awsa

'tortilla': LH eje, MIS aja 'maíz'

'nariz': LH nepsek, LS nepkuru, MA nam-n, CA nam, SU nạ̣-tak, UL nạ̣-tak 'miel': LS wehle 'miel', CA ala 'miel', SU walạ̣ka 'abeja tacaní', UL walạ̣ka 'abeja tacaní', MIS walaṇka 'abeja tacaní'

$\mathrm{PL} / * \mathrm{a} / \mathrm{PM} / * \mathrm{a} /$

'agua': LH was, LS wal, MA was 'lluvia', SU was, UL was

'en': LS k'ati ‘en', SU kat 'en', UL kat 'en', MIS kat 'hasta'

'extremidad': LS kal 'brazo/aleta', CA kari 'brazo', SU kal 'pie', UL kal 'pie', MIS

klahkla 'brazo'

'este': LH na, LS na-num 'aquí', MIS naha

$\mathrm{PL} / *_{\mathrm{O}} / \mathrm{PM} / * \mathrm{a} /$

'excremento': LS po- 'defecar', SU ba, UL ba 'excremento'

'venir': LS po-, MIS bal-

'comer': LH kor-, UL kas-

$\mathrm{PL} / * \mathrm{O} / \mathrm{PM} / * \mathrm{u} /$

'cabeza': LH troh, UL tun

'humo, nube': LS mo 'humo', CA amu 'nube', SU UL MIS mukus 'nube'

'guayabo': LS tororo, CA tur

'olomina' (tipo de pececillo): LS orum, CA urụ̄ni

$\mathrm{PL} / * \mathrm{u} / \mathrm{PM} / * \mathrm{u} /$

'laurel': LS jumam, SU sum, UL sum, MIS sum

'teta, chupar': LS ts'u- 'chupar', ts'ukị̄ 'teta', MA su-m 'teta', CA su-m 'teta', SU su- 'chupar', UL supi 'teta'

'podrirse': LS lukawa, CA duraka, SU dutla, UL dutạ̣

'tierra': LS lu, MA duju, CA duru 


\section{Las listas empleadas para el estudio glotocronológico con identificación de los cognados}

Para el estudio glotocronológico se trató de llenar la lista de 215 rubros (Swadesh 1955) para el cacaopera, el sumo, el ulua, el misquito y las dos lenguas lencas. No se intentó incluir el matagalpa porque el léxico disponible es excesivamente exiguo. Las limitaciones del vocabulario disponible en el caso de las lenguas lencas y el cacaopera determinaron que la lista que se pudo levantar estuviera integrada por 120 rubros solamente. A continuación se da la lista en cuestión. Los cognados se han marcado por medio de la colocación de la misma letra al lado entre paréntesis. Así, en el primer rubro 'agua', los rubros del cacaopera y del misquito aparecen marcados con (a) porque son cognados el uno del otro.

\begin{tabular}{|c|c|c|c|c|c|c|}
\hline & $\mathrm{CA}$ & SU & UL & MIS & LS & $\mathrm{LH}$ \\
\hline agua & li (a) & was (b) & was (b) & li (a) & wal (b) & was (b) \\
\hline amarillo & maju & lalahni (a) & lalahka (a) & lalalhni (a) & $\mathrm{ku}$ & suninga \\
\hline amarrar & wiri- (a) & sit (b) & sit- (b) & wilk- (a) & war- (a) & kup- \\
\hline qquí & iram & akaw (a) & aka (a) & nãhãra (b) & nanum(b) & nawah (b) \\
\hline árbol & $\operatorname{man}(\mathrm{a})$ & pan (a) & $\operatorname{man}(\mathrm{a})$ & dus & sự & ili \\
\hline arena & kui (a) & kawhmak (a) & kawhmak (a) & ) ahuja & mijea & toko \\
\hline ive & wasiri & uhbin & dibaka & pjäwira & ts'imts'im & sira \\
\hline ailar & jaukan & ubu- (a) & abu-/aba:-(a) & dans pul- & uli- (b) & ul- (b) \\
\hline bañar & idiwa- & waska- & wasara- & aihtab- & twa- (a) & twa- (a) \\
\hline eber & dipa (a) & di- (a) & di- (a) & di- (a) & tali- (b) & tal- (b) \\
\hline blanco & sahu (a) & pihni (b) & pihka (b) & pihni (b) & Joko (a) & soko (a) \\
\hline boca & ta-wa (a) & ta-pas (a) & ta-pas (a) & bila & ints'ats'a (b) & in (b) \\
\hline bosque & jura & asạ̣-pas (a) & asaị-pas (a) & unta & kotaṇ (b) & kotan (b) \\
\hline bueno & kua & jamni (a) & jamka (a) & jamni (a) & Jam (a) & sa (a) \\
\hline cabeza & wara & tuna (b) & tuni (b) & lal & oso & $\operatorname{troh}(\mathrm{b})$ \\
\hline caer & kusni & buk- & wauhda- & kahw- & pit- & karau- \\
\hline aliente & dindinka & dajni (a) & dajhka (a) & lapta & $\mathrm{a} \sqrt{\mathrm{a}}$ & siwa \\
\hline aminar & diu- & lapak- & wat- (a) & wap- (a) & o- (a) & $\mathrm{u}-(\mathrm{a})$ \\
\hline amino & lap & ta (a) & ta (a) & jabal & k'in (b) & kin (b) \\
\hline antar & bu- & unbaw- & ajwan- (a) & ajwan- (a) & ihwa- (b) & [门] ]iwa- (b) \\
\hline arne & nakat & di muihni (a) & di muihka (a & )wina (b) & wa $\sqrt{a}(\mathrm{~b})$ & $\mathrm{rah} / \mathrm{rak}$ \\
\hline eniza & wabu (a) & wan (a) & kauh & jampus & pilil & pokko \\
\hline nco & $\begin{array}{l}\text { dukan } \\
\text { panakam }\end{array}$ & sinka (a) & sinka (a) & matsip & ts'aj (b) & saj (b) \\
\hline cola & kuna & mamah & umah & wajka (a) & ts'oh & wah (a) \\
\hline omer & duri & kas- (a) & kas- (a) & pi- & rom- & kor- (a) \\
\hline Smo & maj & ampu (a) & ampat (a) & nahki & ki Ja (b) & kuna (b) \\
\hline & tiwa & karak (a) & karak (a) & wal & tẹ̣ & man \\
\hline rtar & kat- & dak- (a) & dak- (a) & klak- & $t^{\prime}$ ih- (a) & taj- (a) \\
\hline
\end{tabular}




\begin{tabular}{|c|c|c|c|c|c|c|}
\hline corteza & kuta (a) & u-tak (a) & u-tak (a) & taja & ts'ats'a & jos \\
\hline cuatro & butaru & arunka (a) & arunka (a) & walhwal & sa & $\operatorname{aria}(\mathrm{a})$ \\
\hline cuello & tsaw & di-mak (a) & di-t (a) & nana dusa & t'aṇt'aṇ & sala \\
\hline dar & a- & kala- & ja- (a) & jab- (a) & mi- & jar- (a) \\
\hline decir & na- & iti & jul- & wi- & aj- (a) & aj- (a) \\
\hline día & lan & ma (a) & ma (a) & jua & $\mathrm{ja} \sqrt{\mathrm{a}}$ & kasi \\
\hline diente & nini- (a) & ana (b) & ana (b) & na-pa (b) & neh (a) & nek (a) \\
\hline dormir & jabuna (a) & ama-(b) & ama- (b) & jap- (a) & pa- & sar- \\
\hline dos & buru (a) & bu (a) & bu (a) & wal & pe (b) & pe (b) \\
\hline él & karahi (a) & witiọ (b) & alas (a) & witin (b) & inani (c) & ina/inan (c) \\
\hline ellos & karawalhi & witị̄na (a) & alasbalna & witinnani (a) & anani (c) & ananan (c) \\
\hline en & $-\mathrm{m}(\mathrm{a})$ & -kaw (b) & -kaw (b) & $-\mathrm{ra}$ & k'ati (b) & ap (a) \\
\hline esposa & majru (a) & jal (b) & jal (b) & maja (a) & sija & mopa \\
\hline esposo & apu & al (a) & almuk (a) & maja & jiku & asu \\
\hline este & irahi (a) & aka (b) & a:ka (b) & nãhã(c) & inaga (a) & na (c) \\
\hline flor & bas & pulu (a) & pulu (a) & taṛni & Jila (b) & suna (b) \\
\hline frío & tusni & sạ̣ni (a) & ripka & kahula & ts'ana (a) & miti \\
\hline fuego & lawali & kuh (a) & kuh (a) & pawta & ik'aṇ (b) & juka (b) \\
\hline golpear & $\mathrm{du}-$ & wis- & mabaw- & pruk- & $\mathrm{ma} j^{-}-(\mathrm{a})$ & imas- (a) \\
\hline grande & bajaka & nuhni & sikka & ta:ra & puka (a) & puki (a) \\
\hline gusano & bil (a) & babil (a) & witaṇ & liwa & ts'its'a & suru \\
\hline hablar & piswa & jul- (a) & julbaw- (a) & ajs- & Jakini- & mol- \\
\hline hermana & $\mathrm{t} j \mathrm{a}$ & imi- (a) & ami- (a) & lakra & peleh (b) & pela (b) \\
\hline hermano & paj & wahai (a) & wahai(a) & muihni & koh & jokue \\
\hline hígado & kawa & ising (a) & $\operatorname{asing~(a)~}$ & awja & muts’u (b) & musu (b) \\
\hline hoja & taka & bas (a) & bas (a) & waha (b) & aw & wala (b) \\
\hline hombre & misil (a) & al (b) & al (b) & wajtna & $\mathrm{i} j \mathrm{ku}$ & misu (a) \\
\hline hueso & naman & wakal (a) & wakal (a) & dusa & ts'eh (b) & sek (b) \\
\hline humo & kiba (a) & kuh wajaw & b)kuh waju (b) & kjasma (a) & $\operatorname{mo}(c)$ & po:ro (c) \\
\hline largo & wisnika (a) & najni & juhka & wihka (a) & kota- & kasi \\
\hline lavar & saka- (a) & suh- (b) & suh- (b) & tusk- & ts'ih- (a) & sak (a) \\
\hline lejos & ataptika & najkaw & juhka & lajhura & kota (a) & kota (a) \\
\hline lengua & tu-m (a) & tu- (a) & tu- (a) & twisa (a) & nepal (b) & nepel (b) \\
\hline llorar & walwa & ajdi- (a) & ajda- (a) & in- & kaj- & $a j-(a)$ \\
\hline lluvia & ira & was(a) & was (a) & li & fo (b) & so (b) \\
\hline luna & ajku (a) & wajku (a) & wajku (a) & kãti & lets'a & mesti \\
\hline madre & ami (a) & itang (b) & itạ̦ (b) & japti & lạ̣ke & $\operatorname{mina}(\mathrm{a})$ \\
\hline malo & jamua & dutni (a) & dutka (a) & sawra & Jamtoa- & kiauru \\
\hline mano & pana-m & tin- (a) & tin- (a) & mihta & ko\aka(b) & kulala (b) \\
\hline matar & kulina & i- (a) & i- (a) & ik- (a) & Jil- & kas- \\
\hline montaña & karan (a) & asạ̄ (b) & asaụ (b) & asaṇ tara (b) & kotaṇ (a) & kotaṇ (a) \\
\hline morder & api- & kas- (a) & kas- (a) & sam- & ti- & kaj- (a) \\
\hline morir & kula- & daw- & i:wa- & pru- & لil- & kar- \\
\hline mujer & juara (a) & jal (a) & jal (a) & majrin & sija & mop \\
\hline
\end{tabular}




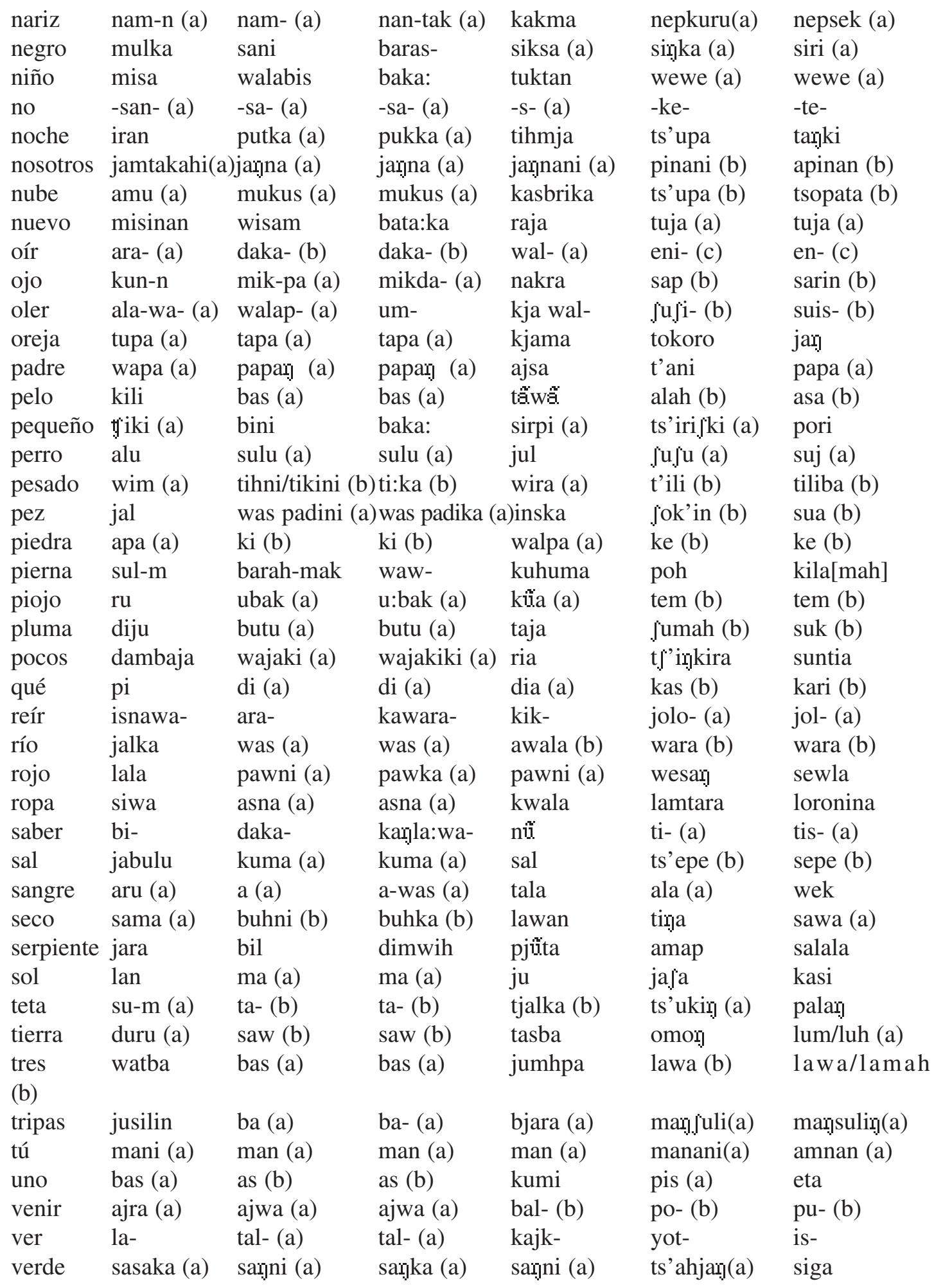




$\begin{array}{lllllll}\text { viejo } & \text { uskam } & \text { umani (a) } & \text { umana (a) } & \text { almuk } & \text { koko } & \text { tahoi- } \\ \text { viento } & \text { win (a) } & \text { win (a) } & \text { win (a) } & \text { pasa } & \text { ujeka } & \text { soror } \\ \text { vientre } & \text { kawa-m (a) ba (b) } & \text { ba (b) } & \text { bjara (b) } & \text { k’omo (a) } & \text { mạ̣kera(b) } \\ \text { vosotros } & \text { maniwalhi(a) manna (a) } & \text { manna (a) } & \text { manani (a) } & \text { kinani } & \text { ajnan } \\ \text { yo } & \text { jami (a) jạ̦ (a) } & \text { jạ̦ (a) } & \text { jạ̦ (a) } & \text { unani (b) } & \text { unan (b) }\end{array}$

\section{Resultados de la aplicación de la glotocronología}

A continuación se dan tres tablas que contienen respectivamente: (a) el número de cognados encontrados en el caso de cada pareja de lenguas, (b) el porcentaje de la lista de 120 rubros que cada número de cognados representa y (c) la separación temporal entre cada pareja de lenguas calculada a partir del respectivo porcentaje. Para el cálculo de la separación temporal se usó la tasa de retención de 86\%, con base en la sugerencia de Hymes (1960: 17) para comprobar indirectamente la adecuación de una tasa a una lista modificada. Este procedimiento consiste en determinar el promedio de los índices de conservación de los rubros particulares que la compongan (índices que se incluyeron en Swadesh 1955) y comparar con los promedios correspondientes de las distintas listas con tasas de retención conocidas, a ver con cuál se da mayor coincidencia. La lista de 120 rubros empleada aquí presenta un promedio de 72,16 , mucho más próximo al de 73,56 de 92 rubros de la lista de 100 con una tasa de retención de $86 \%$ que al de 67,36 de la lista de 215 con una tasa de retención de 80,5\%. Esta aclaración resulta importante en vista de la divergencia que se da entre las separaciones temporales entre las lenguas misumalpas propuestas en este trabajo y en el de Moreira González (1986). Esta autora empleó la tasa de retención de 80,5\% , a pesar de que los promedios correspondientes a sus tres listas son 74,1\%,72,4\% y 70,6\%, lo cual hacía más recomendable el uso de la tasa de $86 \%$.

\section{Tabla 1}

\section{Número de cognados}

$\begin{array}{lcccccc} & \text { CA } & \text { SU } & \text { UL } & \text { MIS } & \text { LS } & \text { LH } \\ \text { CA } & - & 25 & 23 & 18 & 14 & 12 \\ \text { SU } & 25 & - & 92 & 22 & 14 & 16 \\ \text { UL } & 23 & 92 & - & 22 & 13 & 18 \\ \text { MIS } & 18 & 22 & 22 & - & 11 & 13 \\ \text { LS } & 14 & 14 & 13 & 11 & - & 60\end{array}$




\begin{tabular}{lcccccc} 
LH & 12 & 16 & $\begin{array}{c}18 \\
\text { Tabla } 2\end{array}$ & 13 & 60 & - \\
& \multicolumn{7}{c}{ Porcentaje de cognados } \\
& CA & SU & UL & MIS & LS & LH \\
& & & & & \\
CA & - & 20,83 & 19,17 & 15,00 & 11,67 & 10,00 \\
SU & 20,83 & - & 76,67 & 18,33 & 11,67 & 13,33 \\
UL & 19,17 & 76,67 & - & 18,33 & 10,83 & 15,00 \\
MIS & 15,00 & 18,33 & 18,33 & - & 9,17 & 10,83 \\
LS & 11,67 & 11,67 & 10,83 & 9,17 & - & 50,00 \\
LH & 10,00 & 13,33 & 15,00 & 10,83 & 50,00 & -
\end{tabular}

\section{Tabla 3}

\section{Separación temporal entre las lenguas}

$\begin{array}{llllll}\text { CA } & \text { SU } & \text { UL } & \text { MIS } & \text { LS } & \text { LH }\end{array}$

$\begin{array}{ccccccc}\text { CA } & - & 5.195 \pm 542 & 5.470 \pm 569 & 6.281 \pm 658 & 7.113 \pm 742 & 7.625 \pm 791 \\ \text { SU } & 5.195 \pm 542 & - & 880 \pm 166 & 5.616 \pm 582 & 7.113 \pm 742 & 6.672 \pm 692 \\ \text { UL } & 5.470 \pm 569 & 880 \pm 166 & - & 5.616 \pm 582 & 7.361 \pm 755 & 6.282 \pm 659 \\ \text { MIS } & 6.281 \pm 658 & 5.616 \pm 582 & 5.616 \pm 582 & - & 7.914 \pm 838 & 7.361 \pm 755 \\ \text { LS } & 7.113 \pm 742 & 7.113 \pm 742 & 7.361 \pm 755 & 7.914 \pm 838 & - & 2.295 \pm 292 \\ \text { LH } & 7.625 \pm 791 & 6.672 \pm 692 & 6.282 \pm 659 & 7.361 \pm 755 & 2.295 \pm 292 & -\end{array}$

\section{Conclusiones}

En los 93 conjuntos de cognados reunidos en este estudio se dan, como se puede ver en 2., numerosas correspondencias fonológicas sistemáticas entre las lenguas lencas y las misumalpas. La cantidad y la consistencia de estas y el hecho de que los cognados sean en su mayoría términos de vocabulario básico (por ejemplo, 'agua', 'cortar', 'dar', 'diente', 'dónde','dulce', 'en', 'este', 'frío', 'hombre', 'ir', 'lavar', 'nariz', 'padre', 'piedra’, 'río', etc.) prueban, sin lugar a dudas, que hay relación de parentesco entre las dos familias.

La aplicación de la glotocronología que se presenta (que, en el caso de las, lenguas mi- 
sumalpas no incluye al matagalpa) indica que la escisión del protolenca-misumalpa se inició, aproximadamente, hace unos 7180 años (el promedio de las separaciones temporales entre las lenguas misumalpas y las lenguas lencas); la del protomisumalpa en el misquito y el subantepasado de las demás lenguas misumalpas, hace unos 5838 años (el promedio de las separaciones temporales entre el misquito y las otras lenguas misumalpas); la del subantepasado del matagalpa y el cacaopera, y el subantepasado del sumo y el ulua, hace unos 5333 años (el promedio de las separaciones temporales entre el sumo y el ulua con el cacaopera) y la del sumo y el ulua, hace unos 880 años. La del cacaopera y el matagalpa, según los datos de Moreira González (1986), se habría dado hace unos 1218 años. Por su parte, las lenguas lencas se habrían separado hace 2.295 años.

De acuerdo con lo anterior, el establecimiento del protolenca-misumalpa en los territorios en que las lenguas lencas y misumalpas se encontraban en el siglo XVI es muy antiguo, remontándose a lo que Willey denominó Período II de la arqueología de la Baja Centroamérica (8000 a 4000 a.C.) caracterizado por un modo de vida cazador-recolector (Fonseca Zamora 1992: 85).

En el caso del protomisumalpa el inicio de su escisión coincide con el del Período III o del modo de vida igualitario recolector y domesticador especializado (4000 a 1000 a.C.). Es interesante que lo mismo ocurra en el caso de la subagrupación de las lenguas chibchenses que abarca a todas menos el paya (véase Constenla Umaña 1995: 45). Todo lo anterior es incompatible con la concepción de autores como Mason, Jijón y Caamaño y Trimborn, prevaleciente hasta la década de 1970, de que los principales pobladores de origen no mesoamericano del oeste de Panamá, Costa Rica, Nicaragua, Honduras y el oriente de El Salvador fueran producto de penetraciones recientes desde Colombia.

La segunda división, entre el subantepasado del matagalpa y el cacaopera, y el del sumo y el ulua, se habría dado hacia el último tercio del cuarto milenio a.C.

Por lo que respecta a la fecha de separación del matagalpa y el cacaopera que se deriva del porcentaje de cognados obtenido por Moreira González (ídem), hacia el 800 d.C., coincide con el momento que se ha propuesto para el establecimiento del primer pueblo mesoamericano, los chorotegas, en Nicaragua (Fowler 1989: 35).

Finalmente, la fecha de separación de las lenguas lencas propuesta coincide con la época de la ocupación de Quelepa en El Salvador, entre el 400 y el 150 a.C., y se ha planteado que este sitio fue establecido por lencas emigrados de Honduras debido a presiones de población (Sheets 1984: 93-4).

\section{Bibliografía}

Arguedas Cortés, Gilda R. 1987. Los fonemas segmentales del protolenca: reconstrucción comparativa. Tesis de maestría: Universidad de Costa Rica.

Bertoglia Richards, Mafalda. 1988. La lengua cacaopera: fonología y léxico. Tesis de maestría: Universidad de Costa Rica.

Campbell, Lyle. 1976. “The last Lenca”. International Journal of American Linguistics 42 (1): 
73-8.

1979. "Middle American Languages". En Lyle Campbell y Marianne Mithun (comp.), 902-1000.

1979. The languages of Native America: Historical and Comparative Assessment. Austin y Londres: University of Texas Press.

Campbell, Lyle, Anne Chapman y Karen Dakin. 1978. "Honduran Lenca”. International Journal of American Linguistics. 44 (4): 330-2.

Chapman, Anne. 1978. Los lencas de Honduras en el siglo XVI. Tegucigalpa, D.C.: Instituto Hondureño de Antropología e Historia.

CIDCA. 1986. Diccionario elemental miskito-español, español-miskito. Managua: Centro de Investigación y Documentación de la Costa Atlántica.

CIDCA-ITM. 1988. Vocabulario preliminar del ulwa (sumu meridional). Managua: Centro de Investigación y Documentación de la Costa Atlántica, Instituto Tecnológico de Massachussetts.

Constenla Umaña, Adolfo. 1987. "Elementos de fonología comparada de las lenguas misumalpas". Revista de Filología y Lingüística de la Universidad de Costa Rica. 13 (1): 129-62.

1991. Las lenguas del Area Intermedia: introducción a su estudio areal. San José: Editorial de la Universidad de Costa Rica.

1993. “La familia chibcha.” En: María Luisa Rodríguez de Montes (Ed.), pp. 75-125.

1994. "Las lenguas de la Gran Nicoya". Vínculos (Revista Antropológica del Museo Nacional de Costa Rica). 18-19: 191-208.

1995. "Sobre el estudio diacrónico de las lenguas chibchenses y su contribución al conocimiento del pasado de sus hablantes." Museo del Oro (Bogotá) 38-39: 13-55.

Del Río Urrutia, Jimena. 1985. El lenca de Chilanga: fonología y léxico. Tesis de Licenciatura: Universidad de Costa Rica.

Escalante, Roberto y L. Faier. 1959. "Relaciones del Lenca y Xinca”. Ponencia presentada en la reunión anual de la American Anthropological Association.

Fonseca Zamora, Oscar. 1992. Historia antigua de Costa Rica: Surgimiento y caracterización de la primera civilización costarricense. San José: Editorial de la Universidad de Costa Rica. 
Fowler, William R.1989. The Cultural Evolution of Ancient Nahua Civilizations. The PipilNicarao of Central America. University of Oklahoma Press. Norman.

Greenberg, Joseph H. 1987. Language in the Americas . Stanford, California: Stanford University Press.

Herranz, Atanasio. 1987. "El lenca de Honduras: una lengua moribunda". Mesoamérica. 14: 429-66.

Houwald, Götz Von. 1980. Diccionario Español-Sumu, Sumu-Español. Managua: Ministerio de Educación.

Hymes, Dell H. 1960. "Lexicostatistics so far.” Current Anthropology 1 (1): 3-43.

Jijón y Caamaño, Jacinto. 1943. El Ecuador Interandino y Occidental, Vol. 3: Las lenguas del sur de Centro-América y el norte y centro del Oeste de Sud-América. Quito: Editorial Ecuatoriana.

Lange, F. W. y D. Stone (comps.). The Archaeology of Lower Central America: 85-112. Albuquerque, Nuevo México: University of New Mexico Press.

Lehmann, Walter. 1920. Zentral-Amerika . Primera Parte. Die Sprachen Zentral-Amerikas . Tomos 1 y 2. Berlín: Verlag Dietrich Reimer.

Mason, John Alden. 1943. "Idiomas Indígenas y su estudio”. América Indígena. 3: 231-44.

Moreira González, Yamileth. 1986. Análisis lexicoestadístico de las relaciones entre el cacaopera, el matagalpa, el sumo septentrional, el ulua y el misquito. Tesis de licenciatura: Universidad de Costa Rica.

María Luisa Rodríguez de Montes (Ed.). Estado actual de la clasificación de las lenguas indígenas de Colombia, Ponencias presentadas en el Seminario-Taller realizado en el Instituto Caro y Cuervo (Febrero 10, 11 y 12 de 1988). Bogotá: Instituto Caro y Cuervo. Biblioteca "Ezequiel Uricoechea" 11.

Schmidt, Wilhelm. 1926. Die Sprachfamilien und Sprachenkreise der Erde. Winter. Heidelberg.

Sheets, Payton D. 1984 “The Prehistory of El Salvador: An Interpretive Summary”. En: Lange, F. W. y D. Stone (comp.), 85-112.

Swadesh, Morris. 1955. "Towards Greater Accuracy in Lexicostatistic dating”. International Journal of American Linguistics. 21: 121-37. 
1958. "La lingüística de las regiones entre las civilizaciones mesoamericanas." Actas del XXXIII Congreso Internacional de Americanistas.

Willey, Gordon R. 1984. "A Summary of the Archaeology of Lower Central America". En: Lange, F. W. y D. Stone (comps.) 341-78.

Wyllys, Andrew E. 1979. Correspondencias fonológicas entre el lenca y una lengua mayance. San Salvador: Ministerio de Educación. 